\title{
Effect of Physical Training on Exercise-Induced Inflammation and Performance in Mice
}

OPEN ACCESS

Edited by:

Zhichao Fan,

UCONN Health, United States

Reviewed by:

Katsuhiko Suzuki,

Waseda University, Japan

Nan Zhang,

Washington University in St. Louis,

United States

Yuanzhen Suo,

Peking University, China

*Correspondence: Barbara Maximino Rezende

barbaramaximino@ufmg.br;

barbaramaximinorez@gmail.com

Vanessa Pinho

vpinhos@gmail.com

tThese authors have contributed equally to this work

Specialty section:

This article was submitted to Cell Adhesion and Migration,

a section of the journal

Frontiers in Cell and Developmental

Biology

Received: 03 November 2020 Accepted: 18 January 2021

Published: 04 February 2021

Citation:

Barcellos LAM, Gonçalves WA,

Esteves de Oliveira MP,

Guimarães JB, Queiroz-Junior CM,

Resende $C B$, Russo RC,

Coimbra CC, Silva AN, Teixeira MM,

Rezende BM and Pinho V (2021)

Effect of Physical Training on

Exercise-Induced Inflammation

and Performance in Mice.

Front. Cell Dev. Biol. 9:625680.

doi: 10.3389/fcell.2021.625680

\author{
Luiz Alexandre Medrado de Barcellos ${ }^{1 \dagger}$, William Antonio Gonçalves ${ }^{2 \dagger}$, \\ Marcos Paulo Esteves de Oliveira², Juliana Bohnen Guimarães', \\ Celso Martins Queiroz-Junior'2, Carolina Braga de Resende ${ }^{3}$, Remo Castro Russo ${ }^{4}$, \\ Cândido Celso Coimbra ${ }^{4}$, Albená Nunes Silva ${ }^{5}$, Mauro Martins Teixeira ${ }^{6}$, \\ Barbara Maximino Rezende ${ }^{7 * t}$ and Vanessa Pinho ${ }^{2 * t}$
}

' Departamento de Ciências do Movimento Humano, Universidade do Estado de Minas Gerais (UEMG) - Unidade Ibirité, Ibirité, Brazil, ${ }^{2}$ Departamento de Morfologia, Instituto de Ciências Biológicas (ICB), Universidade Federal de Minas Gerais (UFMG), Belo Horizonte, Brazil, ${ }^{3}$ Hospital das Clínicas, Universidade Federal de Minas Gerais (UFMG), Belo Horizonte, Brazil, ${ }^{4}$ Departamento de Fisiologia e Biofísica, Instituto de Ciências Biológicas (ICB), Universidade Federal de Minas Gerais (UFMG), Belo Horizonte, Brazil, ${ }^{5}$ Laboratório de Inflamação e Imunologia do Exercício, Departamento de Educação Física, Escola de Educação Física da Universidade Federal de Ouro Preto, Ouro Preto, Brazil, ${ }^{6}$ Departamento de Bioquímica e Imunologia, Instituto de Ciências Biológicas (ICB), Universidade Federal de Minas Gerais (UFMG), Belo Horizonte, Brazil, 7 Departamento de Enfermagem Básica, Escola de Enfermagem, Universidade Federal de Minas Gerais (UFMG), Belo Horizonte, Brazil

Acute exercise increases the amount of circulating inflammatory cells and cytokines to maintain physiological homeostasis. However, it remains unclear how physical training regulates exercise-induced inflammation and performance. Here, we demonstrate that acute high intensity exercise promotes an inflammatory profile characterized by increased blood IL-6 levels, neutrophil migratory capacity, and leukocyte recruitment to skeletal muscle vessels. Moreover, we found that physical training amplified leukocyteendothelial cell interaction induced by acute exercise in skeletal muscle vessels and diminished exercise-induced inflammation in skeletal muscle tissue. Furthermore, we verified that disruption of the gp-91 subunit of NADPH-oxidase inhibited exerciseinduced leukocyte recruitment on skeletal muscle after training with enhanced exercise time until fatigue. In conclusion, the training was related to physical improvement and immune adaptations. Moreover, reactive oxygen species (ROS) could be related to mechanisms to limit aerobic performance and its absence decreases the inflammatory response elicited by exercise after training.

Keywords: physical training, muscular inflammation, neutrophil, exercise, oxidative stress

\section{INTRODUCTION}

Inflammation is a natural process of mammalian immune responses to tissue damage, triggered by invading pathogens or sterile tissue injury. This response comprises temporally and spatially orchestrated events in which inflammatory cells and mediators act to interrupt harmful stimuli and induce tissue repair, promoting a return to homeostasis (Medzhitov, 2010; Alessandri et al., 2013). Exercise-induced inflammation has been shown to be an essential for restoring homeostasis and improving physical capacity (Deyhle et al., 2015). During exercise, muscle resident cells and vascular endothelium release inflammatory mediators that generate chemotactic signals, which drive leukocyte trafficking to muscular tissue (Kolaczkowska and Kubes, 2013). The presence of these cells is associates with loss of muscular architecture after exercise (Ogilvie et al., 1988; 
Hortobagyi et al., 1998). This effect could be induced by metabolic processes characterized by events involving oxidative stress (Barbieri and Sestili, 2012).

Oxidative stress induced by reactive oxygen species (ROS) is increased by muscular contraction (Jackson et al., 2007) and is able to prolong muscular inflammation induced by acute exercise. Several studies have indicated that ROS produced during the exercise are required to activate signal transduction pathways in inflammation mechanisms and have an essential role in the physiological adaptive process in muscle cells (Barbieri and Sestili, 2012). An increase in antioxidant capacity through the intake of nutritional supplements appears to reduce exerciseinduced muscle damage and may improve performance and recovery (Child et al., 1999; Taherkhani et al., 2020). However, whether training is able to provoke an adaptation in the inflammatory response induced by exercise and whether ROS are important to this process remain unclear. Thus, the present study aimed to assess the influence of physical training on inflammation and physical performance and the role of ROS in this process.

\section{MATERIALS AND METHODS}

\section{Ethics Statement}

The animal care and handling procedures were in accordance with the guidelines of the Institutional Animal Care and Use Committee, and the study received prior approval from Animal Ethics Committee of Universidade Federal de Minas Gerais (UFMG; protocol 7412/2012).

\section{Mice}

Eight to twelve weeks-old wild-type C57BL/6 male mice were obtained from the Centro de Bioterismo (UFMG) and gp91 ${ }^{\text {phox }}$ knockout C57BL/6 male mice were provided from Jackson Farms (Glensville, NJ, United States) and maintained at our laboratory. All mice were housed under standard conditions in a temperature-controlled room $\left(23 \pm 1^{\circ} \mathrm{C}\right)$ on an automatic $12 \mathrm{~h} / 12 \mathrm{~h}$ light/dark cycle and had free access to commercial rodent food and water.

\section{Running Treadmill Familiarization}

Running treadmill familiarization was performed on different treadmills (LE400, Panlab, Harvard Apparatus, Cornella, Spain or Gaustec Magnetismo, Nova Lima, MG, Brazil) according to each design as previous protocols (Lacerda et al., 2005; Primola-Gomes et al., 2007).

\section{Incremental-Speed Running Test Until Fatigue}

An incremental-speed running test until fatigue on a treadmill (LE400, Panlab, Harvard Apparatus, Cornella, Spain) was performed to assess the peak speed $\left(S_{\text {peak }}\right)$. All these incremental running tests were performed at least $24 \mathrm{~h}$ after the last familiarization session. During this test, the mice began running at $6 \mathrm{~m} \mathrm{~min}^{-1}$ and the speed was increased by $3 \mathrm{~m} \mathrm{~min}^{-1}$ every
$3 \mathrm{~min}$ at $5^{\circ}$ slope until fatigue (Ferreira et al., 2007). Fatigue was defined as the moment at which the animals were unable to maintain their pace with the treadmill speed for at least $10 \mathrm{~s}$, even when exposed to slight electrical stimulation.

\section{Rest and Low or High Intensity Exercise}

Wild-type C57BL/6 male mice were allocated into three groups, rest, low intensity $\left(40 \%\right.$ of $\left.\mathrm{S}_{\text {peak }}\right)$, or high intensity $(80 \%$ of $\mathrm{S}_{\text {peak }}$ ) of exercise on the treadmill to analyze oxygen consumption $\left(\mathrm{VO}_{2}\right)$ (LE400, Panlab, Harvard Apparatus, Cornella, Spain). The exercise intensity was calculated from $S_{\text {peak }}$ achieved in the incremental speed-running test until fatigue. Each exercise or rest lasted $30 \mathrm{~min}$. The slope of the treadmill was maintained at $5^{\circ}$. All these procedures were performed at least $48 \mathrm{~h}$ after the incremental-speed running test until fatigue.

\section{Fixed-Speed Running Test Until Fatigue}

The workload and the $\mathrm{VO}_{2}$ of the mice at fixed-speed running test until fatigue on treadmill was evaluated (LE400, Panlab, Harvard Apparatus, Cornella, Spain) pre (at least $48 \mathrm{~h}$ after incrementalspeed running test until fatigue) and post 4 weeks aerobic training (at least $48 \mathrm{~h}$ after the last training session). The intensity of this test corresponded to $80 \%$ of the $S_{\text {peak }}$ achieved at incrementalspeed running test until fatigue and the treadmill slope adopted was also $5^{\circ}$. The workload attained in the fixed-speed running test was used as a reference for the load prescription in the exercise sessions throughout the aerobic training.

\section{Physical Training Protocol}

In all procedures involving training the mice were randomized allocated into sedentary or trained experimental groups. The trained group was submitted to exercise on a treadmill (Gaustec Magnetismo, Nova Lima, MG, Brazil) across 4 weeks, with 5 weekly sessions always performed at the same time of the day (8:00 a.m. to 10:00 a.m.). The slope of the treadmill was maintained at $5^{\circ}$ throughout the training protocol. To ensure similar handling and exposure for each treadmill setup, the sedentary group mice performed an exercise at a speed of 6 $\mathrm{m} \min ^{-1}$ with maximal duration of $5 \mathrm{~min}$, which was adjusted daily according to the body mass of the mice. The aerobic training load from the first to the last week was equivalent to $60,70,80$, and $90 \%$ of the workload (\%W) performed in the fixed-speed running test until fatigue prior to the 4 weeks aerobic training.

\section{$\mathrm{VO}_{2}$ Measurement}

$\mathrm{VO}_{2}$ was measured via open-flow indirect calorimeter (LE400, Panlab, Harvard Apparatus, Cornella, Spain) calibrated with a certified gas mixture (high $\mathrm{O}_{2}=50.05 \%$, high $\mathrm{CO}_{2}=1.51 \%$, low $\mathrm{O}_{2}=20.02 \%$ and low $\left.\mathrm{CO}_{2}=0.00 \%\right)$. The air flow rate established was equivalent to $0.6 \mathrm{~L} \mathrm{~min}^{-1}$ throughout the procedures. $\mathrm{VO}_{2}$ data were analyzed using a computerized system (Metabolism software version 2.2.01 Panlab, Harvard Apparatus), transformed to milliliters per minute and relativized by the mice body mass $\left(\mathrm{mLO}_{2} \mathrm{~kg}^{-0} \cdot{ }^{75} \mathrm{~min}^{-1}\right)$. 


\section{Body Mass}

The percentage of the body mass variation was calculated by the difference between the weight of the mouse before the second test (post training) and the weight before the first test (pre training) divided by the pre training weight, multiplied by 100 .

\section{Assessment of Pulmonary Mechanics}

Pulmonary dysfunction was measured as we previously described (Campa et al., 2018; Russo et al., 2018). For invasive in vivo assessment, mice were anesthetized and tracheostomized, then were placed in a whole-body plethysmograph to maintain spontaneous breathing connected to a computer-controlled ventilator (Forced Pulmonary Maneuver System ${ }^{\circledR}$, Buxco Research Systems $@$, Wilmington, North Carolina, United States). Under mechanical respiration the Dynamic Compliance (Cdyn) and Lung Resistance (Rl) were determined by Resistance and Chord Compliance RC test. To measure the Inspiratory Capacity (IC) a Pressure-Volume maneuver was performed, which inflates the lungs to a standard pressure of $+30 \mathrm{~cm} \mathrm{H}_{2} \mathrm{O}$ and then slowly exhales until a negative pressure of $-30 \mathrm{~cm} \mathrm{H}_{2} \mathrm{O}$ is reached. To evaluate airway hyperresponsiveness (AHR), the same mice used in previous maneuvers (basal condition) received Methacholine, $1 \mathrm{mg} \mathrm{Kg}^{-1}$ (Acetyl- $\beta$-methylcholine chloride, A-2251, Sigma-Aldrich St. Louis, MO, United States) i.v. and after $10 \mathrm{~s}$, a new set of maneuvers were conducted to assess $\mathrm{Rl}$ changes. Suboptimal maneuvers were rejected and for each test at least three acceptable maneuvers were conducted in every single mouse to obtain a reliable mean for all numeric parameters.

\section{Intravital Microscopy}

The mice were anesthetized, and the femoral straight muscle venules were exposed by a resection in the anterior part of the thigh for exhibition of the femoral straight muscle. The animals received an i.v. injection from Rodamin $6 \mathrm{G}\left(0.3 \mathrm{mg} \mathrm{kg}^{-1}\right.$, SigmaAldrich, Germany) to fluorescent labeling of leukocytes. An intravital microscope (ECLIPSE 50i; Nikon) with a 20 objective lens was used to examine the muscle microcirculation. A digital camera (DS-Qi1MC; Nikon) was used to acquire the images that were recorded for playback analysis with Nikon imaging software. The counting of rolling and adherent leukocytes was realized according to our previously published method (Rezende et al., 2017). Rolling leukocytes was defined as those cells moving thought the observed field at a velocity less than that of erythrocytes within a given vessel during $1 \mathrm{~min}$. Leukocyte was considered to be adherent if it remained stationary for at least $30 \mathrm{~s}$, and total leukocyte adhesion is quantified as the number of adherent cells within a $100 \mu \mathrm{m}$ length of venule in $1 \mathrm{~min}$.

\section{Quantification of Cytokines}

Hundred milligrams of the quadriceps muscle (wet) were separated and homogenized with PBS containing antiproteases (0.1 mM PMSF, $0.1 \mathrm{nM}$ hydrochloric benzethonium, $10 \mathrm{mM}$ EDTA and $20 \mathrm{Ki}$ aprotinin A) and $0.05 \%$ Tween 20. The samples were centrifuged for $10 \mathrm{~min}$, at $10,000 \mathrm{rpm}$ and at $4^{\circ} \mathrm{C}$. The supernatant was used for the ELISA assay with a 1: 4 dilution. The ELISA assay was performed according to the manufacturer's instructions (R\&D System) and quantified from the $492 \mathrm{~nm}$ wavelength acquired in a plate reader (Spectramax plus 384, Molecular Devices, United States).

\section{Histology}

Left femoral quadriceps was sectioned transversely in half, with the proximal half placed in O.C.T (Tissue-Tek ${ }^{\circledR}$; Sakura, Netherlands) at $-25^{\circ} \mathrm{C}$. The tissues were sliced at $10 \mu \mathrm{m}$ thickness using a cryostat (Leica CM1850, Leica Biosystems, Germany), immediately placed on silanized slide and fixed for $1 \mathrm{~h}$ in acetone at $-80^{\circ} \mathrm{C}$. The tissue sections were stained with $\mathrm{HE}$ according to standard histological technique and evaluated histologically in a blinded manner. The following parameters were evaluated and classified as absent, mild, moderate, or intense: fiber atrophy (morphological alteration), muscle necrosis/degeneration, inflammatory infiltrate in the endomysium/perimysium, endomysium/perimysium distension. These criteria were first described by Rizo-Roca et al. (2015).

\section{Neutrophil Chemotaxis Assays}

Neutrophil chemotaxis assay was performed using a modified Boyden chamber (Neuroprobe, Pleasanton, United States) and polycarbonate filters (4 $\mu \mathrm{m}$; Neuroprobe, Pleasanton, United States) as previously described by Tavares-Murta et al. (2002). Bone marrow neutrophils were isolated and submitted to chemotactic stimulus with $28 \mu \mathrm{L}$ of $\mathrm{N}$-formyl-methionylleucyl-phenylalanine (fMLP). On one side of the membrane, the fMLP was placed, and on the other, a suspension of $1.0 \times 10^{6}$ neutrophils $/ \mathrm{mL}$. After $60 \mathrm{~min}$ of incubation at $37^{\circ} \mathrm{C}$, the filter was removed, washed and fixed in methanol. Then, the membranes were stained in panotic for microscopic counting. Five random fields per sample were selected on the membrane to count cell migration.

\section{Statistical Analysis}

Data are expressed as mean \pm SEM. The normal distribution was verified by the Shapiro-Wilk test. Comparisons among the groups were performed by unpaired Student's $t$-test, one-way ANOVA or two-way ANOVA followed by the Tukey or Sidak post hoc analysis, whenever applicable. Statistical significance was set as $p<0.05$. The statistical package used was GraphPad Software's Prism $6^{\circledR}$.

\section{RESULTS}

The magnitude and amount of stress induced by movement are important factors for morphological and functional changes in the body. We first determined the exercise intensity required to induce an efficient acute response during a single exercise session consisting of running on a treadmill. We observed that high intensity exercise $\left(80 \%\right.$ of the $\left.S_{\text {peak }}\right)$ triggered an aerobic demand that increased in a time dependent manner. This was observed as a gradual increase in oxygen consumption $\left(\mathrm{VO}_{2}\right)$ during the course of exercise (Figure 1A). Low exercise intensity $\left(40 \%\right.$ of the $\left.\mathrm{S}_{\text {peak }}\right)$ was unable to induce significant changes in $\mathrm{VO}_{2}$ (Figure 1A). Moreover, we found increased muscular levels 

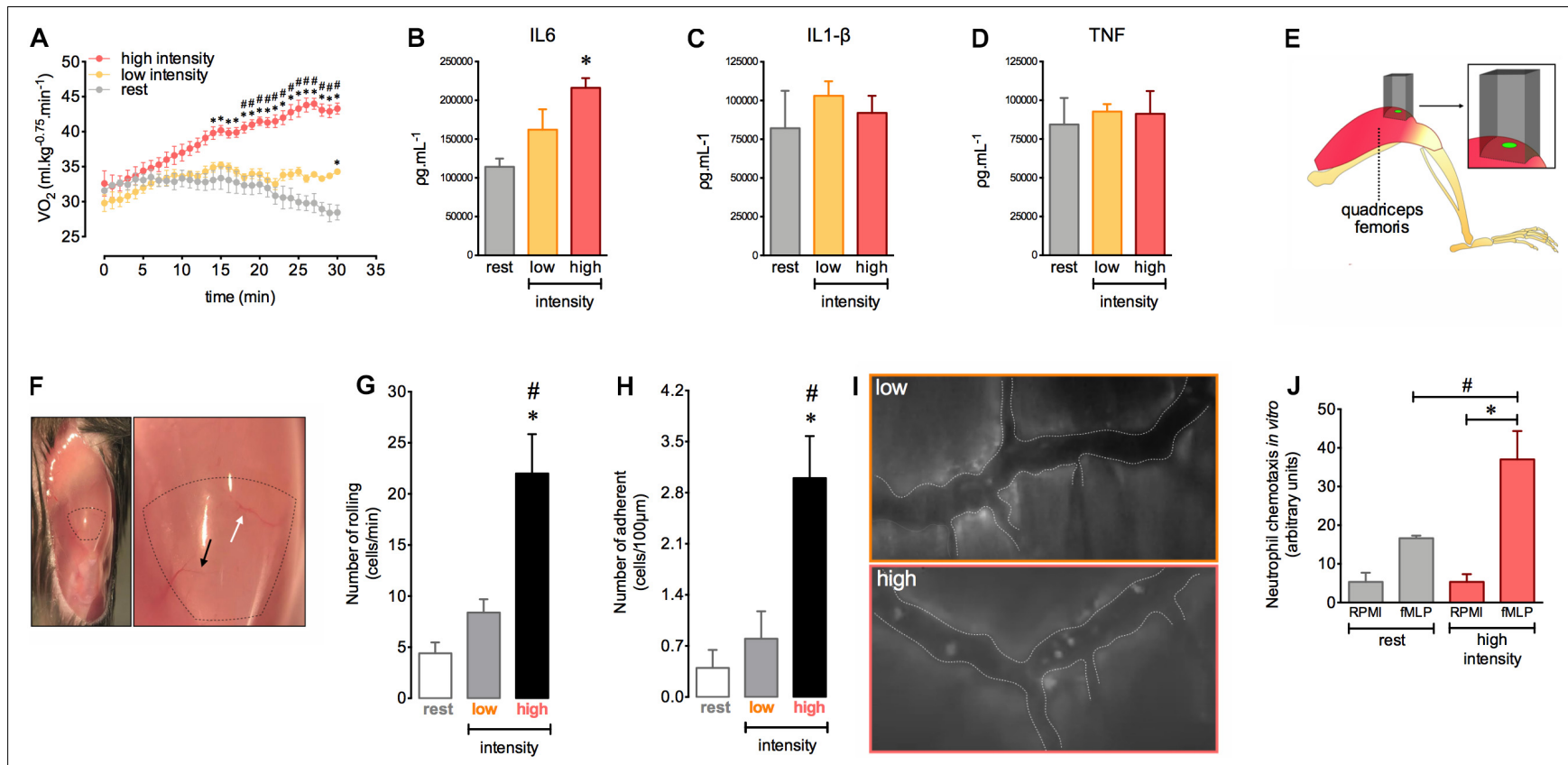

FIGURE 1 | Homeostasis disruption by exercise induces leukocyte recruitment and late alteration of IL-6 balance in quadriceps muscles. VO $\mathrm{O}_{2}$ immediately before and during 30 min at rest $(n=5)$ or at low $(n=5)$ and at high $(n=6)$ intensity exercises (A). IL-6 (B), IL-1 $\beta$ (C), and TNF (D) levels in the quadriceps femoris 12 h after rest $(n=4)$ or low $(n=4)$ and high intensity $(n=4)$ exercises. Neutrophil chemotaxis by Boyden chamber assay. Bone marrow isolated-neutrophils $\left(1 \times 10^{6}\right.$ cells/ml) from rest $(n=3)$ or high intensity $(n=3)$ groups stimulated by fMLP $(28 \mu \mathrm{l})(\mathbf{E})$. The light beam from intravital microscopy targeting the distal region of the quadriceps femoris for intravital analysis (F). Exposed quadriceps for intravital experiment (G). The most (black arrows) and least (white arrows) frequently accessed vessels in the delimited region of the quadriceps. Leukocyte rolling $\mathbf{( H )}$ and adhesion $\mathbf{( I )}$ on microvasculature $12 \mathrm{~h}$ after rest $(n=5)$, low $(n=5)$ or high intensity $(n=6)$. Representative figure showing the vessel (dotted white line) and leukocytes (luminous bodies) quantified in (H-J). Data are expressed as mean $\pm \mathrm{SEM}$; ${ }^{*} p<0.05$ compared to: rest $(\mathbf{A}, \mathbf{B}, \mathbf{H}, \mathbf{I})$ and RPMI high intensity group (E) and ${ }^{\#} p<0.05$ compared to: low intensity group (A,H,I) and fMLP rest group (E) using two-way ANOVA (A) or one-way ANOVA (B-E,H,I) followed by Tukey post hoc analysis.

of IL-6 after $12 \mathrm{~h}$ in mice subjected to high intensity exercise (Figure 1B). The levels of IL1- $\beta$ and TNF- $\alpha$ were similar in all groups (Figures 1C,D).

Given the importance of the immune response during physical training, we next evaluated the interactions between leukocytes and muscle microvasculature after a single exercise session at different intensities. We assessed a specific distal region of the quadriceps femoris, localized near the rectus femoris tendon (Figure 1E). To ensure reproducibility and to reduce variability during measurements, we chose two vessels in this muscle region to better track the vessels during intravital microscopy analysis (Figure 1F). We observed an increase in leukocyte rolling and adhesion in the muscular vessels after $12 \mathrm{~h}$ in the group subjected to high intensity exercise (Figures 1G-I). We also found increased neutrophil chemotaxis toward chemotactic factor fMLP ex vivo using cells purified from the bone marrow of exercised mice (Figure 1J). Together, these results indicate that high intensity exercise applied by running on a treadmill offers the optimal conditions to provoke an important inflammatory response in muscular tissue.

Our experimental design (Figure 2A) was able to induce several adaptations in mice. Of note, the proposed aerobic training protocol offered a gradual increase in the load based on a targeted workload for each training session (Figure 2B, for more details see section "Materials and Methods"). Here, this experimental approach was efficient to elicit consistent change in mice aerobic status. We observed that mice increased the workload and total running distances in aerobic tests after the training period (Figures 2C,D, respectively). Moreover, the body mass variation in trained mice was less pronounced compared to the sedentary group (Figure 2E). These consistent alterations in aerobic status and control of the increase in body mass were accompanied by enhanced lung function in response to stressful exercise. We observed that $72 \mathrm{~h}$ after a single exercise session under fatigue conditions, the sedentary mice group displayed reduced inspiratory capacity and compliance (Cchord) in response to stressful exercise and an increased sensitivity to bronchoconstriction evoked by methacholine injection. However, the trained mice did not present this impact of stressful exercise, showing preserved inspiratory capacity and compliance accompanied by reduced airway hyper reactivity induced by methacholine (Figures $\mathbf{2 F}$ $\mathbf{H}$, respectively). No changes in the basal resistance were observed (Figure 2I).

The aerobic training protocol was able to induce inflammatory response adaptations in muscular tissue. After $12 \mathrm{~h}$ of the fixed-speed running test there was an increase in both rolling and adhesion of leukocytes in muscle vessels of trained mice (Figures 3A-C). Nevertheless, $72 \mathrm{~h}$ after this test the loss in muscular architecture and the increase of inflammatory 


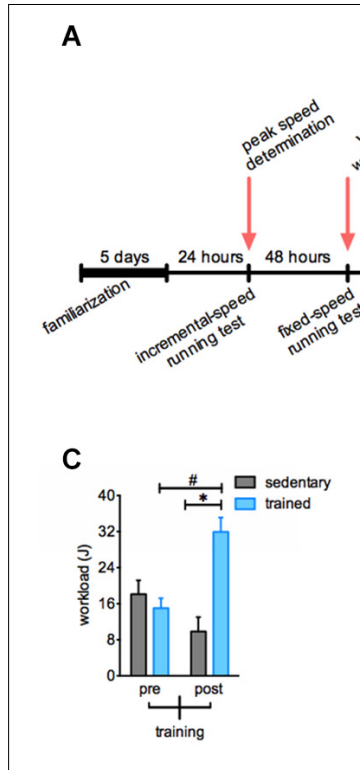

A

D

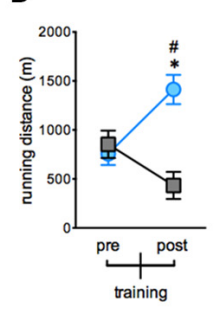

E

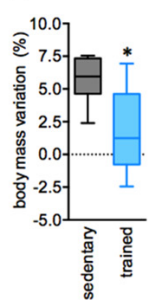

B

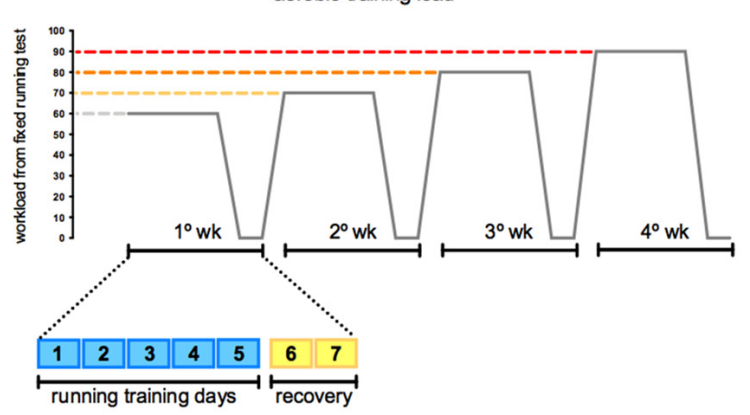

FIGURE 2 | The aerobic training changes body mass gain dynamics, aerobic performance and respiratory mechanics in mice. Schematic diagram for experimental approach visualization (A). Aerobic training load characteristics (B). Workload (C) and running distance (D) evaluated pre and post aerobic training in both sedentary $(n=7)$ and trained $(n=8)$ groups. Body mass variation in sedentary and trained groups $\mathbf{( C )}$. Inspiratory capacity $\mathbf{( F )}$, chord compliance $\mathbf{( G )}$, lung resistance $\mathbf{( H )}$, and basal lung resistance (I) in the naive $(n=8)$, sedentary $(n=6)$ and trained groups $(n=8)$ evaluated $72 \mathrm{~h}$ after the fixed-speed running test until fatigue post training. Data are expressed as mean (C,D,F-I) or min to max in the box plot graph (E) \pm SEM. * $p<0.05$ compared to: sedentary post (C); trained pre (D); sedentary (E-I) and ${ }^{\#} p<0.05$ compared to: trained pre (C); sedentary post (D) using two-way ANOVA following by Sidak post hoc analysis (C,D), two-tailed $t$-test (E) or one-way ANOVA followed by Tukey's post hoc analysis (F-I).

cells were more pronounced in sedentary than trained mice (Figures 3D,E). These results indicated that the profile of inflammatory response induced by exercise exhibited by trained mice could be important to the subsequent recovery of muscular tissue. Moreover, we demonstrated that trained mice presented a slight alteration in cellular profile with an increase in the blood CD8 + lymphocytes and a decrease in the bone marrow macrophages F4/80 + (Supplementary Figures 1D,G, respectively). We not observed any alterations in other leukocytes recovered in either blood (Supplementary Figures 1A-C) or bone marrow (Supplementary Figure 1F,H,I).

To verify the influence of ROS production on aerobic status after training and in leucocyte interactions with the muscular vasculature after exercise, we used gp91phox-/mice, which are animals with non-functional NADPH-oxidase. Trained mice showed a higher $\mathrm{VO}_{2}$ at the moment of fatigue than sedentary mice. Furthermore, it was observed that physical training improved the time to fatigue in both WT and gp91 phox-/- as demonstrated by the higher workload achieved in the fixed-running test compared to the respective sedentary mice. However, exercise interruption post training was delayed for $\mathrm{gp} 91^{\mathrm{phox}-/-}$ trained mice compared to WT trained mice (Figure 4A). In addition, the lack in ROS production potentializes the improvement in the running performance induced by aerobic training, which could be evidenced by the superior increase of workload realized by gp91 phox-/- trained mice compared to workload of WT trained mice (Figure 4B). In contrast, the increase of rolling and adhesion expected $12 \mathrm{~h}$ after a single exercise session was inhibited in trained mice with a deficiency of ROS production (Figures 4C-E).

\section{DISCUSSION}

Although it has been established that a single session of intense exercise is able to induce a considerable local and systemic inflammatory response, which could be reduced by short period of exercise repetition (Suzuki et al., 1996, 1999), little is known about the chronic effects of the training process on exerciseinduced inflammation. The results found in this study can be summarized by the following points: acute high intensity exercise induced an important inflammatory response in the muscular tissue as demonstrated by (1) increases in the level of IL- 6 in muscle, the amplified number of rolling and adhesion cells in the quadriceps vessels, and the stimulation of neutrophil chemotactic activity isolated from bone marrow in exercised mice. Moreover, we developed an effective running training for mice that was able to induce several adaptations, including (2) elevated workload and running distances reached in aerobic tests after the training period accompanied by reduced gains in body mass; (3) enhanced lung function; (4) high number of rolling and adherent cells on muscle vessels, but preserved quadriceps muscular architecture with a predominance of mononuclear cells. Finally, the deficiency in ROS production by NADPH oxidase enzyme (5) potentialized the improvement in the running performance induced by 

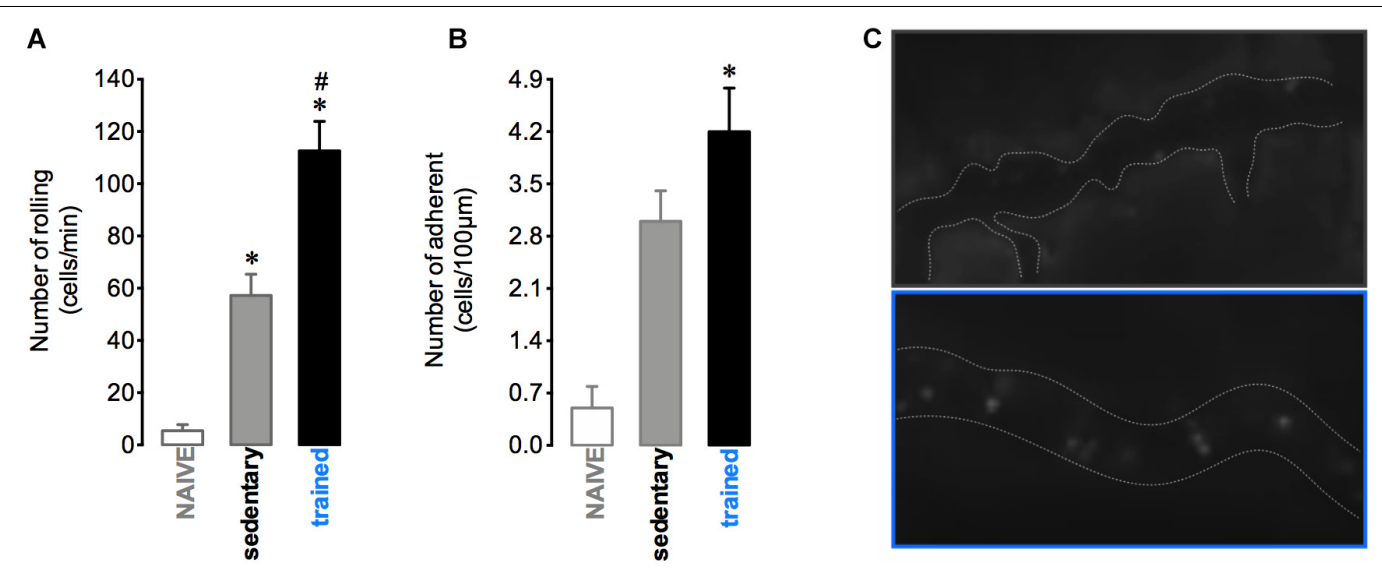

D

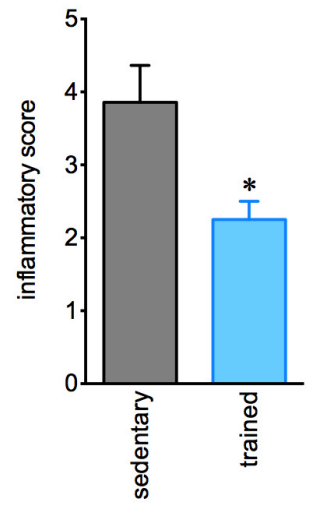

E

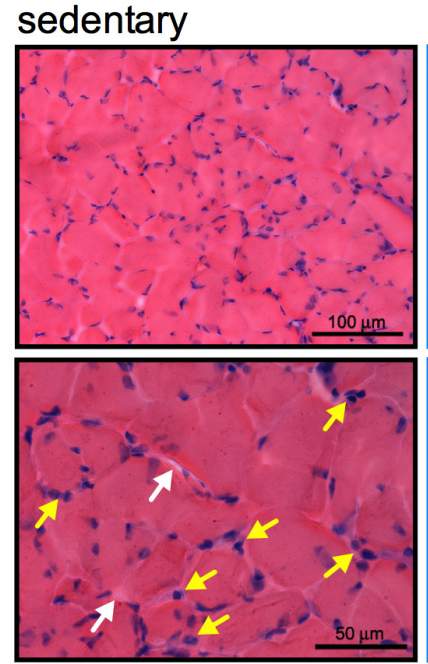

trained
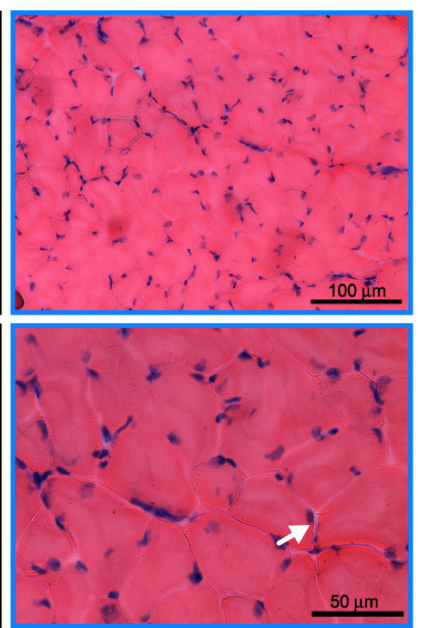

FIGURE 3 | Aerobic training changes the inflammatory response in the muscle. Quantification of leukocyte rolling (A) and adhesion (B) on microvasculature of the naive $(n=6)$, sedentary $(n=5)$, and trained $(n=6)$ mice $12 \mathrm{~h}$ after the fixed-speed running test until fatigue post training. Representative figure showing the vessel (dotted white line) and leukocytes (luminous bodies) quantified in (A-C). Histopathological quantification (D) and representative histological slide from H\&E staining (E) of muscular tissue samples from sedentary $(n=7)$ or trained $(n=5)$ mice $72 \mathrm{~h}$ after the fixed-speed running test until fatigue post training. The yellow and white rows represent the inflammatory cells and endomysium distension, respectively. Data are expressed as mean \pm SEM; ${ }^{*} p<0.05$ compared to: naive (A,B) or sedentary group (D) and ${ }^{\#} p<0.05$ compared to: sedentary group (A) using one-way ANOVA followed by Tukey's post hoc analysis (A,B) or two-tailed $t$-test (C,D).

physical training, and (6) diminished the rolling and adhesion cells related to exercise.

In this work, the intensity of exercise was decisive in stimulating the muscle IL- 6 synthesis assessed $12 \mathrm{~h}$ post exercise. Muscle production of IL-6 is related to the exercise mode, intensity, and duration (Nieman et al., 1998; Ostrowski et al., 2001). Muscle-produced IL-6 may act on skeletal muscle, in a paracrine manner, by regulating myogenesis, glucose uptake and use of glycogen by the contracting muscle and also exert systemic effects such as increased hepatic glucose production and lipolysis, when released into the circulation (Fischer, 2006; Serrano et al., 2008). Circulating IL-6 normally increases immediately in response to contracting muscle and declines in the postexercise period (Suzuki et al., 2002). Raised plasma IL-6 after exercise may a play role in neutrophil mobilization into the circulation (Yamada et al., 2002). In fact, the kinetics of IL6 may be different depending on whether there was muscle damage induced by exercise (Febbraio and Pedersen, 2002; Petersen and Pedersen, 2006). Thus, it is suggested that the highest level of IL-6 in the muscle $12 \mathrm{~h}$ after the exercise event could originate from immune cells present in the damaged muscle during high-intensity exercise. We also observed that acute high intensity exercise induced elevation of the number of rolling and adherent cells in the muscle vasculature. It is well established that prolonged exercise is associated with high levels of oxidative stress that stimulate the inflammatory response. This inflammation may result from muscle damage triggered by mechanical and metabolic exercise loads (Barbieri and Sestili, 2012). Previous notable results from our group also showed the interaction of neutrophils with endothelial cells in 

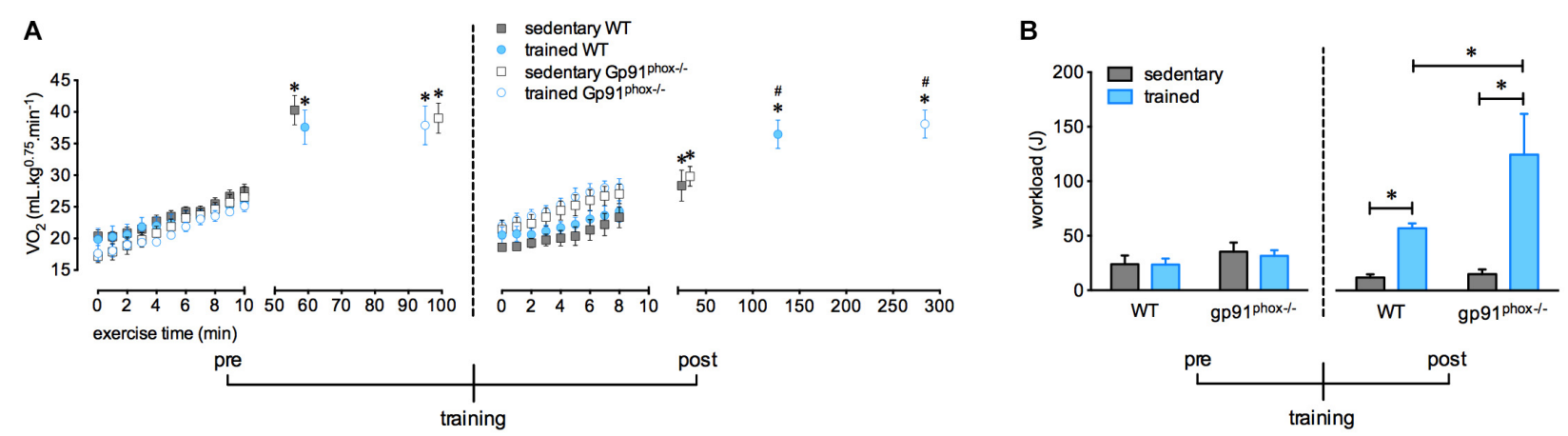

C

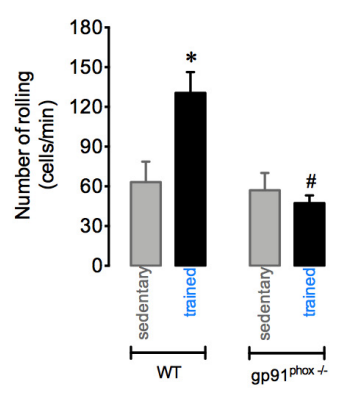

D

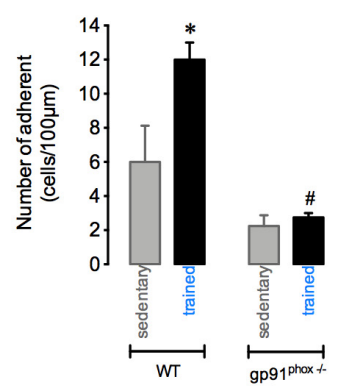

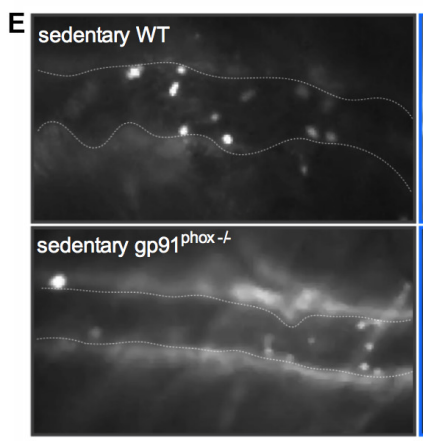

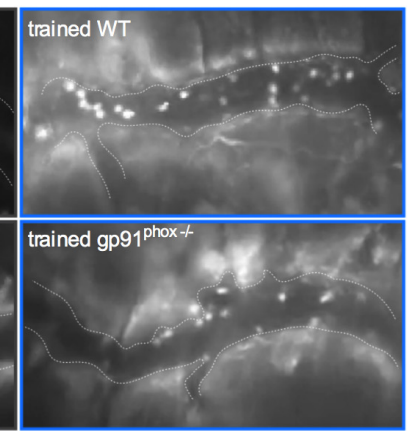

FIGURE 4 | The absence of ROS from NADPH-oxidase increases running capacity and abrogates the greater exercise-induced leukocyte interaction on vasculature after aerobic training. $\mathrm{VO}_{2}$ immediately before and during the fixed-running test until fatigue in the wild-type (sedentary, $n=7$ and trained, $n=6$ ) and gp91 phox-/(sedentary, $n=5$ and trained, $n=4$ ) groups, pre and post aerobic training (A). Workload pre- and post-aerobic training in all groups (B). Leukocytes rolling (C) and adhesion (D) on microvasculature $12 \mathrm{~h}$ after the fixed-speed running test until fatigue in all groups. Representative figure showing the vessel (dotted white line) and leukocytes (luminous bodies) quantified in (C-E). Data are expressed as mean \pm SEM; * $p<0.05$ compared to: 0 min (A) and respective sedentary groups (B-D) and ${ }^{\#} p<0.05$ compared to: respective sedentary $\mathbf{( A )}$ and respective trained groups $(\mathbf{C}, \mathbf{D})$ using two-way ANOVA followed by Tukey's post hoc analysis.

quadriceps muscles after exercise until fatigue by using intravital microscopy, corroborating our findings. We found that high intensity exercise stimulated the ex vivo migratory capacity of neutrophils isolated from bone marrow $12 \mathrm{~h}$ post-exercise. The migration of neutrophils from bone marrow to the circulation is influenced by stress hormones, G-CSF and cytokines released during exercise (Suzuki et al., 2000; Yamada et al., 2002; Summers et al., 2010). Additionally, it has been showed that aerobic exercise mobilizes hematopoietic stem cells in an intensity-dependent manner (Baker et al., 2017). However, the precise mechanisms by which exercise inflammation leads to hematopoiesis and consequently neutrophilia still need to be better elucidated.

We demonstrated an effective protocol of running training for mice that was able to improvement of physical capacity and pulmonary function, including inspiratory capacity, chord compliance, and lung resistance. It has been previously shown that pulmonary capability is correlated with exercise tolerance (Kerti et al., 2018) and it may be associated with our results from the elevated workload and running distances reached in the aerobic tests after the training period.

Interestingly, although we observed a greater number of rolling and adherent cells in trained mice compared to sedentary mice, this result was accompanied by a lower inflammatory score in the quadriceps of the trained group, with a predominance of mononuclear cells. Usually, neutrophils infiltrate the extracellular space around the damage, peaking between 6 and $24 \mathrm{~h}$ after exercise. Subsequently, there is an increase of pro-inflammatory macrophages within $72 \mathrm{~h}$ followed by an influx of macrophages with anti-inflammatory and pro-myogenic phenotypes, which may remain for more than 6 days after damage (Saini et al., 2016). In the present study, reduced muscular inflammation infiltration and endomysium distension indicate less tissue damage, which may be related to mononuclear cells with anti-inflammatory properties, but the profile and role of these cells in muscle repair were not investigated here. Exercise is accepted as an anti-inflammatory therapy in inflammatory diseases such as cardiovascular disease, diabetes, and Alzheimer's (Pedersen and Saltin, 2015). The mechanisms by which exercise training induces these anti-inflammatory effects remains unclear, but there are several intriguing possibilities, including release of endogenous products, such as heat shock proteins, selective reduction of visceral adipose tissue mass or reduction of infiltration of adipocytes by macrophages, shifts in immune cell phenotype, cross-tolerizing effects, or exercise-induced shifts in accessory proteins of toll-like receptor signaling (Petersen and Pedersen, 2006; Flynn et al., 2007). It is also important to assess whether 
the arrival dynamics of cell subtypes is modified by physical training, as monocytes, which could orchestrate inflammation resolution, repair, and increase muscle performance. This possibility deserves further investigation in the future.

In a previous study published by our group pharmacological blockade (apocynin) or genetic deletion of NADPH-oxidase (gp91 ${ }^{\text {phox }-/-}$ mice) inhibited leukocytes recruitment after a single fatiguing exercise. Furthermore, the reduction of ROS production by apocycnin prevented the exercise-induced adhesion molecules (E-selectin, L-selectin and PECAM) expression, which may explain the increasing in the numbers of rolling, adherent, and transmigrating neutrophil, showing the role of ROS from NADPH-oxidase in the skeletal muscle inflammation process (Nunes-Silva et al., 2014). Thus, ROS may regulate cell adhesiveness to the vessel wall of the exercised muscle. ROS- mediated leukocytes recruitment after exercise may also contribute to the muscle remodeling signaling, including angiogenesis, hypertrophic response and mitochondrial biogenesis (Gomes et al., 2012). Similarly, using the genetically modified animals gp $91^{\text {phox }-/-}$, we demonstrated that deficiency of ROS is related to a lower number of rolling and adherent leukocytes in the muscle vessels after training. Moreover, the gp91phox-/- mice presented an exacerbation in the increase of the workload induced by training. This result suggests that the chronic absence of ROS could impair a probable role of exerciseinduced inflammation in controlling fatigue mechanisms and perhaps expose the organism to risks related to excessive exercise. Thus, the importance of investigations on the use of antioxidant strategies for exercise performance is highlighted. In fact, several studies have explored the effectiveness of antioxidant supplementation to enhance performance and adaptation to training, but its benefits and risks remain unknown (Bjornsen et al., 2016; Rothschild and Bishop, 2020).

In conclusion, acute high intensity exercise induced an evident inflammatory response. Additionally, the training was related to physical improvement and immune adaptations. Finally, our data suggest that ROS could be related to maintaining signaling for the limits of aerobic physical performance adaptation to training through the increased inflammatory response elicited by exercise. Nevertheless, the role of oxidative balance on aerobic performance and exercise-induced inflammation after training still needs to be elucidated.

\section{DATA AVAILABILITY STATEMENT}

The original contributions presented in the study are included in the article/Supplementary Material, further inquiries can be directed to the corresponding author/s.

\section{ETHICS STATEMENT}

The animal study was reviewed and approved by Animal Ethics Committee of Universidade Federal de Minas Gerais (UFMG; protocol 7412/2012).

\section{AUTHOR CONTRIBUTIONS}

LB: data collection, analysis of the results, and writing of the manuscript. WG: data collection, writing of the manuscript, and built the figures. $\mathrm{ME}$ and CR: data collection. JG: assisted during interpretation of the results and writing of the manuscript. CQ-J: microscopy analysis and interpretation of the results. RR: pulmonary data collection and interpretation of the results. CC: assisted during the exercise data collection and availability of the laboratory. AS: assisted during the project planning, writing of the manuscript, and the data collection. MT: assisted during the project planning and financial support. BR and VP: assisted during the project planning, data collection, and supervise this manuscript. All authors contributed to the article and approved the submitted version.

\section{FUNDING}

This study was supported by grants from the CNPq (Conselho Nacional de Desenvolvimento Científico e Tecnológico), CAPES (Coordenação de Aperfeiçoamento de Pessoal de Nível Superior), FAPEMIG (Fundação de Amparo à Pesquisa do Estado de Minas Gerais), Pró-Reitoria de Pesquisa da Universidade Federal de Minas Gerais, Pró-Reitoria de Pesquisa da Universidade do Estado de Minas Gerais, and INCT em dengue e interação microrganismo hospedeiro.

\section{ACKNOWLEDGMENTS}

We would like to thank Rosemeire Oliveira and Ilma Marçal for their technical assistance.

\section{SUPPLEMENTARY MATERIAL}

The Supplementary Material for this article can be found online at: https://www.frontiersin.org/articles/10.3389/fcell.2021. 625680/full\#supplementary-material

Supplementary Figure 1 | Effect of aerobic training on the profile of immune cells. The percentage of neutrophil $\mathrm{Ly}_{6 \mathrm{G}}{ }^{+}$, macrophage $\mathrm{F} 480^{+}, \mathrm{TCD}^{+}$and TCD $^{+}$cells of the sedentary $(n=7)$ and training $(n=8)$ mice were evaluated in blood (A-D, respectively) and bone marrow (F-I, respectively) by flow cytometry $72 \mathrm{~h}$ after fixed-speed running test until fatigue post training. Representative dot plots illustrating neutrophil $\mathrm{Ly}_{6 \mathrm{G}}{ }^{+}$, macrophage $\mathrm{F}_{40}{ }^{+}, \mathrm{TCD}^{+}$, and $\mathrm{TCD} 8^{+}$cells of the sedentary (upper panel) and trained (below panel) mice in the blood (E) and bone marrow $\mathbf{( J )}$. Data are expressed as mean \pm SEM; * $p<0.05$ compared to sedentary using two-tailed $t$-test. Cells from bone marrow and blood were plated, $1 \times 10^{6}$ cells/well, in a 96-well plate and stained for extracellular molecular expression patterns using mAbs against mouse CD3 (Alexa-488 conjugated), CD4 (APC conjugated), CD8 (PE conjugated), CD11b (Alexa-488 conjugated), F4/80 (PE conjugated), and Ly6G (APC conjugated) (from BD Pharmingen, Le Pont de Claix, France). The cells were incubated with $20 \mu \mathrm{l} /$ well of antibody solution $30 \% / 4^{\circ} \mathrm{C}$, followed by fixation in $4 \%$ of paraformaldehyde. Limits for the quadrant markers were always set based on negative populations and isotype control antibodies. The frequency of positive cells was analyzed by FlowJo X v10.2 software, using a gate that included lymphocytes, neutrophils and/or macrophages. The frequency (percentage) of the analyzed population in the total acquired events was used in the construction of the graphs. 


\section{REFERENCES}

Alessandri, A. L., Sousa, L. P., Lucas, C. D., Rossi, A. G., Pinho, V., and Teixeira, M. M. (2013). Resolution of inflammation: mechanisms and opportunity for drug development. Pharmacol. Ther. 139, 189-212. doi: 10.1016/j.pharmthera. 2013.04.006

Baker, J. M., Nederveen, J. P., and Parise, G. (2017). Aerobic exercise in humans mobilizes HSCs in an intensity-dependent manner. J. Appl. Physiol. 122, 182-190. doi: 10.1152/japplphysiol.00 696.2016

Barbieri, E., and Sestili, P. (2012). Reactive oxygen species in skeletal muscle signaling. J. Signal. Transduct. 2012:982794.

Bjornsen, T., Salvesen, S., Berntsen, S., Hetlelid, K. J., Stea, T. H., LohneSeiler, H., et al. (2016). Vitamin C and E supplementation blunts increases in total lean body mass in elderly men after strength training. Scand. J. Med. Sci. Sports 26, 755-763. doi: 10.1111/sms.1 2506

Campa, C. C., Silva, R. L., Margaria, J. P., Pirali, T., Mattos, M. S., Kraemer, L. R., et al. (2018). Inhalation of the prodrug PI3K inhibitor CL27c improves lung function in asthma and fibrosis. Nat. Commun. 9:5232.

Child, R., Brown, S., Day, S., Donnelly, A., Roper, H., and Saxton, J. (1999). Changes in indices of antioxidant status, lipid peroxidation and inflammation in human skeletal muscle after eccentric muscle actions. Clin. Sci. 96, 105-115. doi: 10.1042/cs0960105

Deyhle, M. R., Gier, A. M., Evans, K. C., Eggett, D. L., Nelson, W. B., Parcell, A. C., et al. (2015). Skeletal muscle inflammation following repeated bouts of lengthening contractions in humans. Front. Physiol. 6:424. doi: 10.3389/fphys. 2015.00424

Febbraio, M. A., and Pedersen, B. K. (2002). Muscle-derived interleukin-6: mechanisms for activation and possible biological roles. FASEB J. 16, 13351347. doi: 10.1096/fj.01-0876rev

Ferreira, J. C., Rolim, N. P., Bartholomeu, J. B., Gobatto, C. A., Kokubun, E., and Brum, P. C. (2007). Maximal lactate steady state in running mice: effect of exercise training. Clin. Exper. Pharmacol. Physiol. 34, 760-765. doi: 10.1111/ j.1440-1681.2007.04635.x

Fischer, C. P. (2006). Interleukin-6 in acute exercise and training: what is the biological relevance? Exerc. Immunol. Rev. 12, 6-33.

Flynn, M. G., Mcfarlin, B. K., and Markofski, M. M. (2007). The anti-inflammatory actions of exercise training. Am. J. Lifestyle Med. 1, 220-235.

Gomes, E. C., Silva, A. N., and De Oliveira, M. R. (2012). Oxidants, antioxidants, and the beneficial roles of exercise-induced production of reactive species. Oxid. Med. Cell Longev. 2012:756132.

Hortobagyi, T., Houmard, J., Fraser, D., Dudek, R., Lambert, J., and Tracy, J. (1998). Normal forces and myofibrillar disruption after repeated eccentric exercise. J. Appl. Physiol. 84, 492-498. doi: 10.1152/jappl.1998. 84.2.492

Jackson, M. J., Pye, D., and Palomero, J. (2007). The production of reactive oxygen and nitrogen species by skeletal muscle. J. Appl. Physiol. 102, 1664-1670. doi: 10.1152/japplphysiol.01102. 2006

Kerti, M., Balogh, Z., Kelemen, K., and Varga, J. T. (2018). The relationship between exercise capacity and different functional markers in pulmonary rehabilitation for COPD. Int. J. Chron. Obstruct. Pulmon. Dis. 13, 717-724. doi: 10.2147/copd. s153525

Kolaczkowska, E., and Kubes, P. (2013). Neutrophil recruitment and function in health and inflammation. Nat. Rev. Immunol. 13, 159-175. doi: 10.1038/ nri3399

Lacerda, A. C., Marubayashi, U., and Coimbra, C. C. (2005). Nitric oxide pathway is an important modulator of heat loss in rats during exercise. Brain Res. Bull. 67, 110-116. doi: 10.1016/j.brainresbull.2005. 06.002

Medzhitov, R. (2010). Inflammation 2010: new adventures of an old flame. Cell 140, 771-776. doi: 10.1016/j.cell.2010. 03.006

Nieman, D. C., Nehlsen-Cannarella, S. L., Fagoaga, O. R., Henson, D. A., Utter, A., Davis, J. M., et al. (1998). Influence of mode and carbohydrate on the cytokine response to heavy exertion. Med.
Sci. Sports Exerc. 30, 671-678. doi: 10.1097/00005768-199805000-0 0005

Nunes-Silva, A., Bernardes, P. T., Rezende, B. M., Lopes, F., Gomes, E. C., Marques, P. E., et al. (2014). Treadmill exercise induces neutrophil recruitment into muscle tissue in a reactive oxygen species-dependent manner: an intravital microscopy study. PLoS One 9:e96464. doi: 10.1371/journal.pone.00 96464

Ogilvie, R. W., Armstrong, R. B., Baird, K. E., and Bottoms, C. L. (1988). Lesions in the rat soleus muscle following eccentrically biased exercise. Am. J. Anat. 182, 335-346. doi: 10.1002/aja.1001820405

Ostrowski, K., Rohde, T., Asp, S., Schjerling, P., and Pedersen, B. K. (2001). Chemokines are elevated in plasma after strenuous exercise in humans. Eur. J. Appl. Physiol. 84, 244-245. doi: 10.1007/s00421017 0012

Pedersen, B. K., and Saltin, B. (2015). Exercise as medicine - evidence for prescribing exercise as therapy in 26 different chronic diseases. Scand. J. Med. Sci. Sports 25(Suppl. 3), 1-72. doi: 10.1111/sms. 12581

Petersen, A. M., and Pedersen, B. K. (2006). The role of IL-6 in mediating the antiinflammatory effects of exercise. J. Physiol. Pharmacol. 57(Suppl. 10), 43-51. doi: 10.1249/00005768-200605001-00226

Primola-Gomes, T. N., Pires, W., Rodrigues, L. O., Coimbra, C. C., Marubayashi, U., and Lima, N. R. (2007). Activation of the central cholinergic pathway increases post-exercise tail heat loss in rats. Neurosci. Lett. 413, 1-5. doi: 10.1016/j.neulet.2006.10.042

Rezende, B. M., Athayde, R. M., Goncalves, W. A., Resende, C. B., Teles De Toledo Bernardes, P., Perez, D. A., et al. (2017). Inhibition of 5-lipoxygenase alleviates graft-versus-host disease. J. Exp. Med. 214, 3399-3415.

Rizo-Roca, D., Rios-Kristjansson, J. G., Nunez-Espinosa, C., Ascensao, A., Magalhaes, J., Torrella, J. R., et al. (2015). A semiquantitative scoring tool to evaluate eccentric exercise-induced muscle damage in trained rats. Eur. J. Histochem. 59:2544.

Rothschild, J. A., and Bishop, D. J. (2020). Effects of dietary supplements on adaptations to endurance training. Sports Med. 50, 25-53. doi: 10.1007/s40279019-01185-8

Russo, R. C., Savino, B., Mirolo, M., Buracchi, C., Germano, G., Anselmo, A., et al. (2018). The atypical chemokine receptor ACKR2 drives pulmonary fibrosis by tuning influx of CCR2(+) and CCR5(+) IFNgamma-producing gammadeltaT cells in mice. Am. J. Physiol. Lung Cell Mol. Physiol. 314, L1010-L1025.

Saini, J., Mcphee, J. S., Al-Dabbagh, S., Stewart, C. E., and Al-Shanti, N. (2016). Regenerative function of immune system: modulation of muscle stem cells. Age. Res. Rev. 27, 67-76. doi: 10.1016/j.arr.2016. 03.006

Serrano, A. L., Baeza-Raja, B., Perdiguero, E., Jardi, M., and Munoz-Canoves, P. (2008). Interleukin-6 is an essential regulator of satellite cell-mediated skeletal muscle hypertrophy. Cell Metab. 7, 33-44. doi: 10.1016/j.cmet.2007. 11.011

Summers, C., Rankin, S. M., Condliffe, A. M., Singh, N., Peters, A. M., and Chilvers, E. R. (2010). Neutrophil kinetics in health and disease. Trends Immunol. 31, 318-324. doi: 10.1016/j.it.2010.05.006

Suzuki, K., Naganuma, S., Totsuka, M., Suzuki, K. J., Mochizuki, M., Shiraishi, M., et al. (1996). Effects of exhaustive endurance exercise and its one-week daily repetition on neutrophil count and functional status in untrained men. Int. J. Sports Med. 17, 205-212. doi: 10.1055/s-2007-97 2833

Suzuki, K., Nakaji, S., Yamada, M., Totsuka, M., Sato, K., and Sugawara, K. (2002). Systemic inflammatory response to exhaustive exercise, Cytokine kinetics. Exerc. Immunol. Rev. 8, 6-48.

Suzuki, K., Totsuka, M., Nakaji, S., Yamada, M., Kudoh, S., Liu, Q., et al. (1999). Endurance exercise causes interaction among stress hormones, cytokines, neutrophil dynamics, and muscle damage. J. Appl. Physiol. 87, 1360-1367. doi: 10.1152/jappl.1999.87.4.1360

Suzuki, K., Yamada, M., Kurakake, S., Okamura, N., Yamaya, K., Liu, Q., et al. (2000). Circulating cytokines and hormones with immunosuppressive but neutrophil-priming potentials rise after endurance exercise in humans. Eur. J. Appl. Physiol. 81, 281-287. doi: 10.1007/s0042100 50044 
Taherkhani, S., Suzuki, K., and Castell, L. (2020). A short overview of changes in inflammatory cytokines and oxidative stress in response to physical activity and antioxidant supplementation. Antioxidants 9:886. doi: 10.3390/antiox90 90886

Tavares-Murta, B. M., Zaparoli, M., Ferreira, R. B., Silva-Vergara, M. L., Oliveira, C. H., Murta, E. F., et al. (2002). Failure of neutrophil chemotactic function in septic patients. Crit. Care Med. 30, 1056-1061. doi: 10.1097/00003246200205000-00017

Yamada, M., Suzuki, K., Kudo, S., Totsuka, M., Nakaji, S., and Sugawara, K. (2002). Raised plasma G-CSF and IL-6 after exercise may play a role in neutrophil mobilization into the circulation. J. Appl. Physiol. 92, 1789-1794. doi: 10.1152/ japplphysiol.00629.2001
Conflict of Interest: The authors declare that the research was conducted in the absence of any commercial or financial relationships that could be construed as a potential conflict of interest.

Copyright $\odot 2021$ Barcellos, Gonçalves, Esteves de Oliveira, Guimarães, QueirozJunior, Resende, Russo, Coimbra, Silva, Teixeira, Rezende and Pinho. This is an open-access article distributed under the terms of the Creative Commons Attribution License (CC BY). The use, distribution or reproduction in other forums is permitted, provided the original author(s) and the copyright owner(s) are credited and that the original publication in this journal is cited, in accordance with accepted academic practice. No use, distribution or reproduction is permitted which does not comply with these terms. 